\title{
Myrtaceae na Bacia do Rio Caveiras: Características Ecológicas e Usos Não Madeireiros
}

\author{
Juliano Pereira Gomes ${ }^{1}$, Helen Michels Dacoregio ${ }^{1}$, Karina Montibeller da Silva ${ }^{1}$, \\ Luiara Heerdt da Rosa ${ }^{1}$, Roseli Lopes da Costa Bortoluzzi ${ }^{1}$
}

${ }^{1}$ Departamento de Engenharia Florestal, Universidade do Estado de Santa Catarina - UDESC, Lages/SC, Brasil

\begin{abstract}
RESUMO
Objetivou-se descrever o padrão florístico-estrutural, as características ecológicas e os potenciais das Myrtaceae na bacia hidrográfica do Rio Caveiras. As comunidades de Myrtaceae estudadas localizam-se no Planalto Sul Catarinense, Lages, São José do Cerrito e Urupema. Foram instaladas quatro blocos por município, totalizando $30 \mathrm{mil} \mathrm{m}^{2}$ de área amostral, nos quais todos os espécimes de Myrtaceae com diâmetro à altura do peito (DAP) $\geq 5 \mathrm{~cm}$ foram amostrados. A estrutura da comunidade de Myrtaceae foi avaliada pelos descritores fitossociológicos e a suficiência amostral foi verificada utilizando-se o método de rarefação. As abordagens ecológicas e indicações de uso não madeireiro basearam-se em bibliografia especializada e base de dados científicos. Foram amostrados 1.480 exemplares de Myrtaceae pertencentes a 21 espécies e 11 gêneros. As espécies mais abundantes foram Myrceugenia euosma, Siphoneugena reitzii e Myrcia palustris, as quais representaram mais de $50 \%$ da estrutura da comunidade. Quanto à classificação ecológica, destacou-se o grupo das secundárias iniciais (57\%). As espécies amostradas são indicadas para restauração de áreas alteradas, usos ornamentais (100\%) e fitoterápicos (60\%). Apesar da representatividade e potencialidades das Myrtaceae, ainda são necessárias pesquisas para embasar a conservação via plano de manejo.
\end{abstract}

Palavras-chave: classificação ecológica, restauração de áreas alteradas, uso fitoterápico.

\section{Myrtaceae in the Caveiras River Basin: Ecological Characteristics and Non-timber Uses}

\begin{abstract}
This study aimed to describe the floristic-structural pattern, ecological characteristics and potentials of Myrtaceae in the Caveiras River Basin. The studied Myrtaceae communities are localized in Santa Catarina Southern Plateau - municipalities of Lages, São José do Cerrito and Urupema. Four sampling units were installed in each municipality, totaling a sampling area of $30,000 \mathrm{~m}^{2}$. All Myrtaceae individuals with $\mathrm{DBH} \geq 5 \mathrm{~cm}$ were surveyed within the sampling units. The Myrtaceae community structure was assessed through phytosociological descriptors and the sampling sufficiency was verified using the rarefaction method. The ecological approach, as well as the indication of non-timber use of Myrtaceae resources was inferred from a specialized bibliography and scientific database. 1,480 Myrtaceae individuals were sampled, totaling 21 species and 11 genera. The most abundant species were Myrceugenia euosma, Siphoneugena reitzii and Myrcia palustris, which represent more than $50 \%$ of the community structure. Regarding the ecological classification, the late secondary group was the most expressive (57\%). The sampled
\end{abstract}


species are recommended for the restoration of altered areas, ornamental (100\%) as well as phytotherapic use (60\%). Despite the Myrtaceae representativeness and potentials, further research is necessary to support conservation through management plans.

Keywords: ecological classification, restoration of altered areas, phytotherapic use.

\section{INTRODUÇÃO}

Florestas nativas subtropicais são conhecidas por desempenharem funções ecológicas essenciais e produzirem expressiva variedade de recursos, entretanto converter produtos florestais em bens e serviços sem alterar o equilíbrio ambiental envolve um grande desafio. As florestas têm sido utilizadas, especialmente, para obtenção de derivados madeireiros, enquanto vasta gama de outros potenciais, como frutífero, oleífero, resinífero, ornamental e medicinal ainda são pouco explorados. A utilização de produtos florestais não madeireiros (PFNM) é uma forma de uso que proporciona a valoração da floresta, a qual é conservada in loco, mantendo a diversidade genética e garantindo recursos às comunidades envolvidas (Torres, 2001).

Dentre as famílias botânicas com expressiva indicação de uso não madeireiro destacam-se Myrtaceae (Camlofski, 2008). Essa família botânica possui distribuição natural por todos os continentes do hemisfério sul, compreendendo 145 gêneros e 5.970 espécies (The Plant List, 2013). No Brasil, sua riqueza é representada por 1.025 espécies, pertencentes a 23 gêneros, ocorrendo, especialmente, na Floresta Atlântica, ecossistema considerado centro de diversidade da família (Sobral et al., 2016).

Dentre as características relevantes das Myrtaceae destacam-se os potenciais melífero (Lughadha \& Proença, 1996) e frutífero (Landrum \& Kawasaki, 1997), pois seus frutos são suculentos e carnosos, alimentando a fauna silvestre, como aves, roedores, macacos, morcegos e peixes (Barroso et al., 1999). Essa interação entre os dispersores e as Myrtaceae favorece a manutenção de serviços ambientais, estimulando o processo de regeneração natural, o que caracteriza essa família com potencial para revegetação de áreas alteradas (Backes \& Irgang, 2002). Além dos importantes serviços ecológicos nos ecossistemas naturais, os frutos das Myrtaceae também são amplamente indicados para consumo humano, pois apresentam propriedades nutracêuticas, pela presença de compostos secundários fitoterápicos, aromáticos e antioxidantes (Limberger et al., 2001; Cardoso et al., 2009; Carvalho et al., 2014).

Devido às importantes particularidades das Myrtaceae nativas, torna-se necessário o conhecimento da composição florística e estrutural das comunidades dessa família, especialmente na Floresta Ombrófila Mista, ambiente em que se destaca pela elevada diversidade (Ferreira et al., 2012; Ferreira et al., 2013; Higuchi et al., 2013). A partir desse conhecimento base é possível a elaboração de planos de manejo e conservação de uma das famílias botânicas mais promissoras para região serrana de Santa Catarina.

Assim, objetivou-se descrever o padrão florísticoestrutural, as características ecológicas e os potenciais de comunidades de Myrtaceae que compõem a flora de remanescentes da Floresta Ombrófila Mista na bacia hidrográfica do Rio Caveiras, Planalto Sul Catarinense.

\section{MATERIAL E MÉTODOS}

\section{1. Área de estudo}

As comunidades de Myrtaceae estudadas localizam-se em remanescentes florestais situados no Planalto Sul Catarinense, nos municípios de Lages, São José do Cerrito e Urupema (Tabela 1), pertencentes à bacia hidrográfica do Rio Caveiras. No município de Urupema, a área estudada destina-se à conservação e nos demais municípios as áreas são utilizadas para agricultura e pecuária.

A classificação de solo foi realizada segundo o dr. Jaime Antônio de Almeida (dados não publicados), especialista em Gênese, Morfologia e Classificação dos Solos.

O clima da região é classificado, segundo Köppen (1948), como Cfb, clima mesotérmico subtropical úmido, com verões frescos, sem estação seca, com geadas severas frequentes e temperaturas médias dos meses mais quentes inferiores a $22{ }^{\circ} \mathrm{C}$ e pluviosidade média entre 1.300 e $1.400 \mathrm{~mm} / \mathrm{ano}$. 
Tabela 1. Características gerais de três municípios abrangidos pela bacia hidrográfica do Rio Caveiras, Planalto Sul Catarinense.

Table 1. General characteristics of three municipalities containing the Caveiras River Watershed, Santa Catarina Southern Plateau.

\begin{tabular}{|c|c|c|c|}
\hline \multirow{2}{*}{ Características } & \multicolumn{3}{|c|}{ Municípios } \\
\hline & Lages & São José do Cerrito & Urupema \\
\hline Coordenadas & $27^{\circ} 51^{\prime} 18^{\prime \prime} \mathrm{S} 50^{\circ} 09^{\prime} 06^{\prime \prime} \mathrm{W}$ & $27^{\circ} 43^{\prime} 25^{\prime \prime} \mathrm{S} 50^{\circ} 39^{\prime} 40^{\prime \prime} \mathrm{W}$ & $27^{\circ} 55^{\prime} 09^{\prime \prime S} 49^{\circ} 51^{\prime} 57^{\prime \prime} \mathrm{W}$ \\
\hline Altitude (m) & 1018 & 798 & 1502 \\
\hline Área (ha) & 123 & 65 & 1.367 \\
\hline Tipo de solo & $\begin{array}{l}\text { Neossolo Litólico Húmico; } \\
\text { Cambissolo Húmico }\end{array}$ & Nitossolo Vermelho & $\begin{array}{l}\text { Neossolo Litólico; } \\
\text { Cambissolo Húmico }\end{array}$ \\
\hline
\end{tabular}

\subsection{Levantamento e análise dos dados}

Em cada município foram alocados quatro blocos com dimensões de $50 \times 50 \mathrm{~m}$, subdivididos em parcelas de $10 \times 10 \mathrm{~m}$, totalizando $30 \mathrm{mil} \mathrm{m}^{2}$ de área amostral. Foram amostrados todos os exemplares arbóreos e arbustivos pertencentes às Myrtaceae com diâmetro à altura do peito (DAP) $\geq 5 \mathrm{~cm}$. Todos os espécimes com estruturas reprodutivas foram coletados. O material botânico foi identificado e classificado com o auxílio de bibliografia, consulta a especialista (Marcos Sobral) e, posteriormente, herborizado e incorporado ao acervo do Herbário Lages da Universidade do Estado de Santa Catarina (LUSC). Os binômios foram conferidos pela Lista da Flora do Brasil (Sobral et al., 2016).

A estrutura horizontal da comunidade das Myrtaceae foi verificada pelos descritores fitossociológicos (densidade absoluta, dominância absoluta e frequência absoluta). Foi avaliada a suficiência amostral pela curva espécie-área, utilizando-se o método de rarefação, aferida segundo Kersten \& Galvão (2011). Todas as análises foram feitas com auxílio do programa estatístico R (versão 2.2.1, R Development Core Team, 2010).

Quanto à abordagem ecológica foram verificadas as fenofases e classificação dos grupos funcionais utilizando-se como base a Flora Ilustrada Catarinense (Legrand \& Klein 1967, 1969a, b, 1970, 1971, 1977, 1978), A Família das Myrtaceae do Rio Grande do Sul e a Flora Digital do Rio Grande do Sul e Santa Catarina. Foram consideradas pioneiras as espécies referidas em literatura (FLORA ILUSTRADA CATARINENSE) como exigentes em luz (heliófitas obrigatórias) e por colonizar orlas e florestas em restauração natural; secundárias iniciais as descritas como heliófilas não obrigatórias, pouco frequentes em matas desenvolvidas; secundárias tardias as espécies seletivas em sombra (esciófitas) que se desenvolvem, especialmente, em submata desenvolvida. Também foram consideradas as observações pessoais a campo. Com relação à nomenclatura dos grupos ecológicos, adotou-se Gandolfi et al. (1995): as espécies foram diferenciadas em pioneiras $(\mathrm{Pi})$, secundárias iniciais $(\mathrm{Si})$ e secundárias tardias (St). A partir da lista florística das Myrtaceae, realizou-se revisão bibliográfica das informações dos potenciais da família em bases de dados científicos (Scielo e Periódicos Capes) e livros.

\section{RESULTADOS E DISCUSSÃO}

Nos remanescentes estudados foram amostrados 1.480 indivíduos de Myrtaceae pertencentes a 21 espécies e 11 gêneros. A amostragem foi suficiente para representar a composição florística da família na área estudada, conforme a tendência de estabilização da curva e aferição de acordo com Kersten \& Galvão (2011) (Figura 1).

O gênero com maior riqueza foi Myrcia (6 espécies), seguido por Myrceugenia (4) e Eugenia (3). Oito gêneros apresentaram apenas uma espécie cada (Tabela 2).

O gênero Myrcia, além de ser o mais rico, também foi o que mais contribuiu para estrutura da comunidade de Myrtaceae, com 509 indivíduos, representando 34,4\% da abundância amostrada. Myrceugenia e Eugenia contribuíram com $19,9 \%$ e $16,7 \%$, respectivamente. Esses três gêneros abrangeram $61,9 \%$ das espécies amostradas e $71 \%$ dos indivíduos de Myrtaceae. Esses expressivos valores de riqueza e abundância também foram relatados por outros autores que realizaram estudos na Floresta Ombrófila Mista (Martins et al., 2011; Higuchi et al., 2012, 2013). 


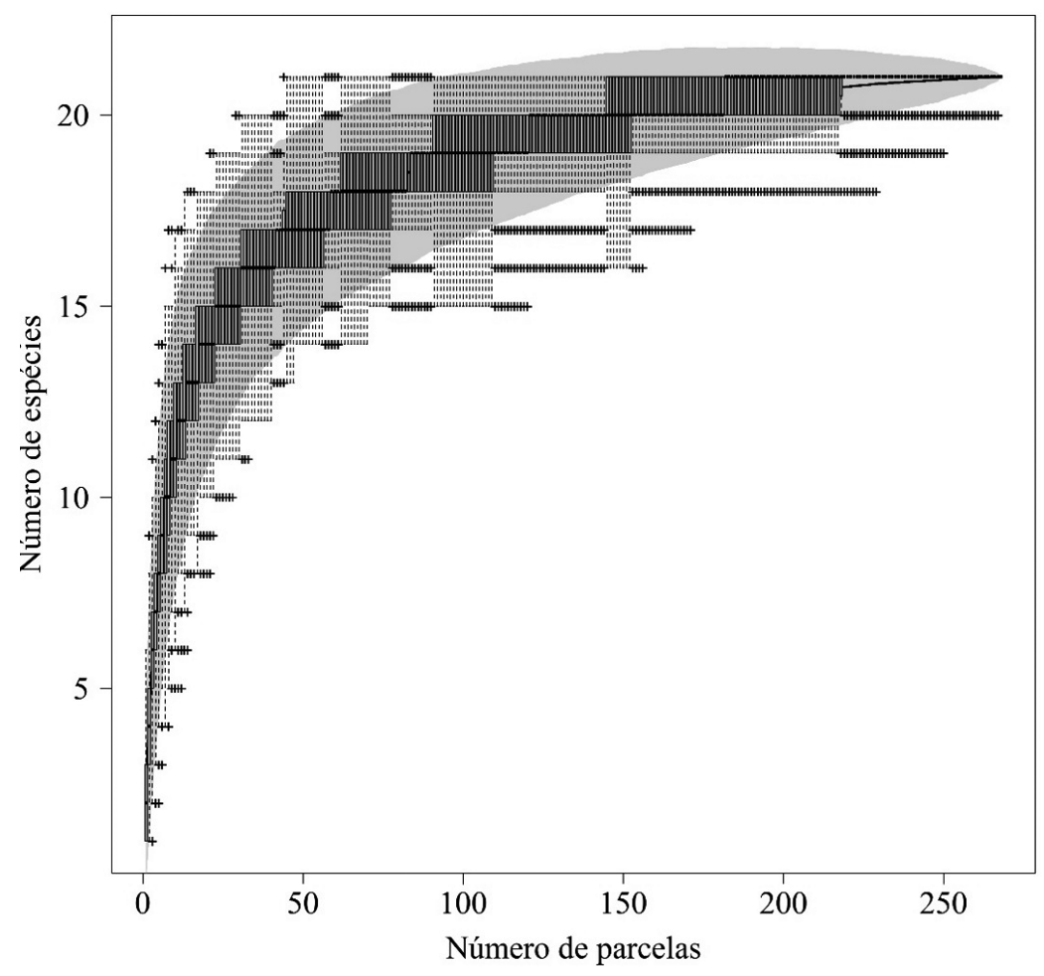

Figura 1. Curva espécies/área usando o método de rarefação das unidades amostrais, da comunidade de Myrtaceae na Bacia Hidrográfica do Rio Caveiras, Planalto Sul Catarinense.

Figure 1. Curve species-area using the rarefation method of sample units, the Myrtaceae community in Basin Caveiras River, Planalto Sul, Santa Catarina.

As espécies mais abundantes (Myrceugenia euosma, Siphoneugena reitzii e Myrcia palustris) totalizaram 760 indivíduos, representando mais de $50 \%$ da estrutura das comunidades de Myrtaceae amostradas ao longo da bacia hidrográfica do Rio Caveiras. Esse fato se deve a coincidência de algumas unidades amostrais terem alocação em sítios propícios à ocorrência dessas espécies, sendo esses fragmentos caracterizados fitofisionomicamente pela elevada abundância de guamirins e cambuís, assim como descrito por Legrand \& Klein (1977), onde são frequentemente representados pelos gêneros Myrceugenia, Myrcia, Myrciaria e Siphoneugena.

Para as espécies amostradas, a floração foi mais expressiva nos meses de novembro, dezembro, janeiro e fevereiro (final da primavera e verão), contudo, nos demais meses do ano, essa fenofase foi representada por ao menos uma espécie de Myrtaceae (Tabela 2). Com relação à frutificação, ressalta-se que em todos os meses do ano apresentaram-se espécies frutificando, sendo que o pico de fornecimento de frutos ocorre nas estações primavera e verão. Essa plasticidade em relação à presença de frutos nas quatro estações possibilita a funcionalidade do sistema, em contínua interação proporcionada pela fauna, assim como observado por Gressler et al. (2006).

Das 21 espécies identificadas, 1 pertence ao grupo ecológico das pioneiras (5\%), 12 , às secundárias iniciais (57\%), e 8, às secundárias tardias (38\%) (Tabela 3). Apesar dos locais amostrados pertencerem a floresta secundária, nota-se que, a partir das Myrtaceae amostradas, está ocorrendo a evolução sucessional, ou seja, está ocorrendo substituição por espécies de estágios funcionais mais avançados.

No grupo das pioneiras, Myrcia oblongata foi a única representante amostrada, entretanto apresentou elevada densidade populacional. É considerada uma das Myrtaceae mais importantes para expansão dos capões de Floresta Ombrófila Mista sobre os campos, pois é tolerante a luminosidade e apresenta rusticidade frente às condições adversas de solo. Essas características 
Tabela 2. Componentes arbóreo-arbustivos das Myrtaceae presentes em remanescentes de Floresta Ombrófila Mista na bacia hidrográfica do Rio Caveiras, Planalto Sul Catarinense, com respectivos descritores fitossociológicos: DA - densidade absoluta; DoA - dominância absoluta; FA - frequência absoluta.

Table 2. The present tree and shrub component of Myrtaceae in remnants of the Mixed Ombrophilous Forest, Caveiras River Watershed, Santa Catarina Southern Plateau, with its respective phytosociological descriptors: DA-absolute density, DoA-absolute dominance, FA-absolute frequency and Phenophases.

\begin{tabular}{|c|c|c|c|c|c|}
\hline \multirow{2}{*}{ Espécies } & \multirow{2}{*}{ DA } & \multirow{2}{*}{ DoA } & \multirow{2}{*}{ FA } & \multicolumn{2}{|c|}{ Fenofases } \\
\hline & & & & Floração & Frutificação \\
\hline Myrceugenia euosma (O. Berg) D. Legrand & 287 & 9,413 & 42 & Set-Fev ${ }^{\mathrm{f}-\mathrm{i}}$ & Jul-Out ${ }^{\mathrm{i}, \mathrm{k}} / \mathrm{Fev}^{\mathrm{d}}$ \\
\hline Siphoneugena reitzii D. Legrand & 244 & 4,375 & 42 & Ago $^{\mathrm{k}} /$ Nov-Maio ${ }^{\mathrm{a}, \mathrm{i}, \mathrm{k}}$ & Out-Dez ${ }^{\mathrm{a}, \mathrm{i}, \mathrm{k}}$ \\
\hline Myrcia palustris DC. & 229 & 3,313 & 67 & Out-Mar ${ }^{\mathrm{g}, \mathrm{i}, \mathrm{k}}$ & Jan-Outg ${ }^{\mathrm{g}, \mathrm{i}, \mathrm{k}}$ \\
\hline Eugenia pluriflora DC. & 161 & 1,128 & 50 & Out-Mar ${ }^{\mathrm{b}, \mathrm{i}, \mathrm{k}}$ & Out-Jan ${ }^{\mathrm{b}, \mathrm{i}, \mathrm{k}}$ \\
\hline Myrcia guianensis (Aubl.) DC. & 130 & 2,632 & 33 & Set-Jan ${ }^{\mathrm{h}, \mathrm{k}}$ & 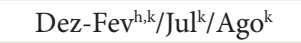 \\
\hline Myrcia oblongata DC. & 122 & 0,716 & 33 & Nov-Fev ${ }^{\mathrm{h}, \mathrm{i}, \mathrm{k}}$ & $\mathrm{Fev}^{\mathrm{h}, \mathrm{i}} / \mathrm{Abr}^{\mathrm{k}} / \mathrm{Jun}^{\mathrm{i}} / \mathrm{Ago}^{\mathrm{d}}$ \\
\hline Eugenia pyriformis Cambess. & 65 & 0,981 & 58 & Nov-Fev ${ }^{b, k}$ & $\mathrm{Jan}^{\mathrm{k}}-\mathrm{Fev}^{\mathrm{b}} / \mathrm{Abr}^{\mathrm{i}}$ \\
\hline Calyptranthes concinna DC. & 57 & 0,461 & 67 & Ago-Feve, ${ }^{\mathrm{i}, \mathrm{k}, \mathrm{k}}$ & Out-Dez $/ J u l^{i}-S_{e}{ }^{k}$ \\
\hline Myrcianthes gigantea (Legr.) Legrand & 44 & 0,733 & 50 & Out-Dez ${ }^{\mathrm{a}, \mathrm{k}}$ & $\operatorname{Jan}^{\mathrm{a}} / \mathrm{Fev}^{\mathrm{i}} / \mathrm{Set}^{\mathrm{k}} / \mathrm{Nov}^{\mathrm{k}}$ \\
\hline Campomanesia xanthocarpa (Mart.) O. Berg & 33 & 1,068 & 33 & Set-Nov ${ }^{\mathrm{a}, \mathrm{k}}$ & Set-Jan ${ }^{a, i, k} / M^{2} a^{i}$ \\
\hline Myrcia laruotteana Cambess. & 23 & 0,124 & 58 & Nov-Jan ${ }^{\mathrm{h}}$ & $\mathrm{Fev}^{\mathrm{d}}$ \\
\hline Acca sellowiana (O. Berg) Burret & 22 & 0,164 & 58 & Out-Jan ${ }^{\mathrm{a}, \mathrm{i}, \mathrm{k}}$ & $\mathrm{Fev}^{\mathrm{k}}-\mathrm{Abr}^{\mathrm{a}-\mathrm{i}}$ \\
\hline Eugenia uruguayensis Cambess. & 21 & 0,225 & 33 & Ago-Mar ${ }^{\mathrm{b}, \mathrm{i}, \mathrm{k}}$ & Abr-Jun ${ }^{\mathrm{k}} /$ Set-Dez ${ }^{\mathrm{b}, \mathrm{i}, \mathrm{k}}$ \\
\hline Blepharocalyx salicifolius Legrand & 19 & 0,584 & 33 & Out-Fev $v^{c, k}$ & Nov-Maioc ${ }^{\mathrm{c}, \mathrm{k}, \mathrm{k}}$ \\
\hline Myrrhinium atropurpureum Schott & 7 & 0,025 & 17 & Mark/Ago-Nova ${ }^{\mathrm{a}-\mathrm{i}}$ & Ago-Maio ${ }^{\mathrm{a}, \mathrm{i}, \mathrm{k}}$ \\
\hline Myrceugenia myrcioides (Cambess.) O. Berg & 4 & 0,091 & 17 & Out $^{\mathrm{k}} / \mathrm{Dez}-\mathrm{Març}^{\mathrm{f}, \mathrm{k}} / \operatorname{Jun}^{\mathrm{k}}$ & Dez $^{k}$ \\
\hline $\begin{array}{l}\text { Myrceugenia oxysepala (Burret) D. Legrand } \\
\text { e Kausel }\end{array}$ & 3 & 0,084 & 17 & Fev-Julf ${ }^{\mathrm{fk}}$ & Julk/Out-Nover,k \\
\hline Myrciaria delicatula (DC.) Berg & 3 & 0,059 & 17 & Dez-Abrc,k/Jun $/$ Set $^{\mathrm{k}}$ & $\mathrm{Nov}^{\mathrm{c}}-\mathrm{Dez}^{\mathrm{k}} / \mathrm{Jun}-\mathrm{Set}^{\mathrm{j}} / \mathrm{Mar}^{\mathrm{k}}$ \\
\hline Myrcia hatschbachii D. Legrand & 3 & 0,044 & 8 & Dez $^{\mathrm{k}}-J_{a n}^{\mathrm{h}}$ & $\mathrm{Fev}^{\mathrm{i}, \mathrm{k}}$ \\
\hline Myrcia hartwegiana (O. Berg) Kiaersk & 2 & 0,004 & 8 & $\mathrm{Mar}^{\mathrm{k}} / \mathrm{Ago}^{\mathrm{k}} / \mathrm{Nov}-\mathrm{Abr}^{\mathrm{g}, \mathrm{k}}$ & Jun $/$ /Out-Dez \\
\hline Myrceugenia glaucescens (Cambess.) & 1 & 0,003 & 8 & Out-Fev ${ }^{\mathrm{f}, \mathrm{k}}$ & $\mathrm{Fev}^{\mathrm{i}, \mathrm{k}}$ \\
\hline
\end{tabular}

${ }^{\mathrm{a}}$ Legrand \& Klein (1977); ${ }^{\mathrm{b}}$ Legrand \& Klein (1969a); 'Legrand \& Klein (1978); ${ }^{\mathrm{d} O b s e r v a c ̧ a ̃ o ~ p e s s o a l ; ~}{ }^{\mathrm{C}}$ Legrand \& Klein (1971); ${ }^{\mathrm{f}} \mathrm{Legrand}$

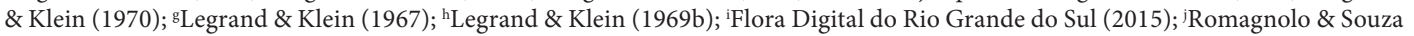
(2004); ${ }^{k}$ Sobral (2003).

favorecem sua indicação para restauração de áreas com distúrbios de maior intensidade. Nessa espécie, um dos componentes químicos majoritários no óleo essencial é o linalol (Henriques et al., 1997), composto muito utilizado pela indústria da alta perfumaria (Letizia et al., 2003) que o considera um ingrediente floral insubstituível. Além disso, pesquisas evidenciam diversas atividades farmacológicas desse componente, inclusive ação antimicrobiana (Park et al., 2012), anti-inflamatória (Huo et al., 2013), antidepressiva (Guzmán-Gutierrez et al., 2012) anticonvulsionante (Elisabetsky et al., 1999) e vasorrelaxante (Anjos et al., 2013).

Com relação às secundárias iniciais, caracterizadas por permanecerem no dossel e/ou subdossel da floresta devido a tolerância à radiação direta, destacam-se
Mirceugenia euosma, Siphoneugena reitzii, Myrcia palustris, Eugenia pluriflora e Myrcia guianensis por apresentarem os maiores valores demográficos na área estudada (Tabela 2). No remanescente localizado no município de Urupema, as espécies M. euosma e S. reitzii, típicas da mata nebular, foram as mais abundantes. De acordo com as características das espécies desse grupo, sugere-se o seu uso nos estágios iniciais e subsequentes, de restauração ou enriquecimento, já que são espécies heliófitas e que apresentam bom desenvolvimento quando em contato com a luz direta.

Dentre as secundárias tardias, encontram-se Calyptranthes concinna, Eugenia uruguayensis, Myrceugenia glaucescens, M. myrcioides, M. oxysepala, Myrcianthes gigantea, Myrciaria delicatula e Myrrhinium atropurpureum, as quais são espécies ciófitas que 
Tabela 3. Uso não madeireiro e grupo ecológico das espécies de Myrtaceae amostradas em três remanescentes de Floresta Ombrófila Mista pertencentes à bacia hidrográfica do Rio Caveiras, Planalto Sul Catarinense. GE = Grupo ecológico; $\mathrm{AU}=$ Arborização urbana; RAA = Recomposição de áreas alteradas; FR = Frutífero; FITO = Fitoterápico; $\mathrm{Pi}=$ Pioneiras; $\mathrm{Si}=$ Secundárias iniciais; $\mathrm{St}=$ Secundárias tardias. A presença de X sem numeração corresponde a observação dos autores.

Table 3. Use non-timber and ecological group of the Myrtaceae species surveyed within the three remnants of Ombrophilous Mixed Forest, which belong to the Caveiras River Watershed, Santa Catarina Southern. Where: $\mathrm{GE}=$ Ecological group; $\mathrm{AU}=$ Urban afforestation; RAA = Recomposition of altered areas; FR = Fructiferous; FITO = Phytotherapic; $\mathrm{Pi}=$ Pioneers; $\mathrm{Si}=$ Early secondary and $\mathrm{St}=$ Late secondary. The presence of $\mathrm{X}$ without numbering corresponds to the author's observations.

\begin{tabular}{|c|c|c|c|c|c|}
\hline \multirow{2}{*}{ Espécies } & \multicolumn{5}{|c|}{ Uso não madeireiro } \\
\hline & GE & AU & RAA & FR & FITO \\
\hline Acca sellowiana (O. Berg) Burret & $\mathrm{Si}$ & $\mathrm{X}^{3,4}$ & $\mathrm{X}^{3,4}$ & $\mathrm{X}^{1,4,3}$ & $\mathrm{X}^{2,5}$ \\
\hline Blepharocalyx salicifolius Legrand & $\mathrm{Si}$ & $\mathrm{X}^{6,7}$ & $\mathrm{X}^{6}$ & & $\mathrm{X}^{2,5}$ \\
\hline Calyptranthes concinna DC. & $\mathrm{Si}$ & $\mathrm{X}$ & $\mathrm{X}^{6}$ & & $\mathrm{X}^{2}$ \\
\hline Campomanesia xanthocarpa (Mart.) O. Berg & $\mathrm{Si}$ & $\mathrm{X}^{4}$ & $\mathrm{X}^{4}$ & $\mathrm{X}^{6,3,7}$ & $\mathrm{X}^{2,5}$ \\
\hline Eugenia pluriflora DC. & $\mathrm{Si}$ & $\mathrm{X}^{8}$ & $\mathrm{X}^{8}$ & & $\mathrm{X}^{2}$ \\
\hline Eugenia pyriformis Cambess. & $\mathrm{Si}$ & $\mathrm{X}^{4,7}$ & $\mathrm{X}^{4,7}$ & $\mathrm{X}^{4,7,9}$ & $\mathrm{X}^{5}$ \\
\hline Eugenia uruguayensis Cambess. & St & $\mathrm{X}$ & $\mathrm{X}$ & & \\
\hline Myrcia guianensis (Aubl.) DC. & $\mathrm{Si}$ & $\mathrm{X}$ & $\mathrm{X}$ & & \\
\hline Myrcia hatschbachii D. Legrand & St & $\mathrm{X}$ & $\mathrm{X}$ & & \\
\hline Myrcia hartwegiana (O. Berg) Kiaersk & $\mathrm{Si}$ & $\mathrm{X}$ & $\mathrm{X}$ & & \\
\hline Myrcia laruotteana Cambess. & St & $\mathrm{X}$ & $\mathrm{X}$ & & \\
\hline Myrcia oblongata DC. & $\mathrm{Pi}$ & $\mathrm{X}$ & $\mathrm{X}$ & & \\
\hline Myrcia palustris DC. & $\mathrm{Si}$ & $\mathrm{X}^{7}$ & $\mathrm{X}^{7}$ & & \\
\hline Myrceugenia euosma (O. Berg) D. Legrand & $\mathrm{Si}$ & $\mathrm{X}^{7}$ & $\mathrm{X}^{6}$ & & $\mathrm{X}^{2}$ \\
\hline Myrceugenia glaucescens (Cambess.) & St & $\mathrm{X}$ & $\mathrm{X}$ & & $\mathrm{X}^{2}$ \\
\hline Myrceugenia myrcioides (Cambess.) O. Berg & $\mathrm{Si}$ & $\mathrm{X}$ & $\mathrm{X}$ & & $\mathrm{X}^{2}$ \\
\hline Myrceugenia oxysepala (Burret) D. Legrand e Kausel & St & $\mathrm{X}$ & $\mathrm{X}$ & & $\mathrm{X}^{2}$ \\
\hline Myrcianthes gigantea (Legr.) Legrand & St & $\mathrm{X}$ & $\mathrm{X}$ & & \\
\hline Myrciaria delicatula (DC.) Berg & St & $\mathrm{X}^{10}$ & $\mathrm{X}$ & $\mathrm{X}^{8,10}$ & \\
\hline Siphoneugena reitzii D. Legrand & $\mathrm{Si}$ & $\mathrm{X}$ & $\mathrm{X}$ & & $\mathrm{X}^{2}$ \\
\hline Myrrhinium atropurpureum Schott & St & $\mathrm{X}$ & $\mathrm{X}$ & & $\mathrm{X}^{2}$ \\
\hline
\end{tabular}

${ }^{1}$ Ducroquet et al. (2000); ${ }^{2}$ Souza et al. (2011); ${ }^{3}$ Legrand \& Klein (1977); ${ }^{4}$ Lorenzi (1998a); ${ }^{5}$ Rio Grande do Sul (2001); ${ }^{6}$ Lorenzi (1998b); ${ }^{7}$ Marchiori \& Sobral (1997); ${ }^{8}$ Lorenzi (2009); ${ }^{9}$ Legrand \& Klein (1969a); ${ }^{10}$ Legrand \& Klein (1978).

preferem solos úmidos e profundos nas submatas dos pinhais (Reitz, 1965). Nenhuma espécie pertencente a essa categoria funcional apresentou expressiva abundância, o que demonstra a necessidade de um conjunto de ações para conservação in situ e ex situ dessas espécies, possibilitando o enriquecimento de projetos de restauração em estágios sucessionais avançados.

Todas as Myrtaceae amostradas podem ser utilizadas para arborização urbana, pois se destacam pela arquitetura de copa, porte (grande parte compõe o subdossel e apresenta crescimento final inferior a 10 metros de altura), floração abundante e frutos de tamanho médio a pequeno.
Além do potencial ecológico e paisagístico, aproximadamente $60 \%$ das Myrtaceae inventariadas possuem potenciais ou usos fitoterápicos comprovados (Tabela 3), fato atribuído à presença de óleos essenciais em seus órgãos, principalmente nas folhas. $\mathrm{O}$ caráter fitoterápico é um importante aliado na conservação das Myrtaceae nativas, pois vincula aspectos econômicos e sociais.

Algumas espécies pertencentes ao gênero Eugenia apresentam atividade anti-inflamatória, analgésica, antipirética, antifúngica, sendo usadas no tratamento de úlceras peptídicas (Kuskoski et al., 2003; Mahmoud et al., 2001). Além disso, muitas espécies do gênero são relatadas como plantas ricas em polifenóis derivados de 
ácido gálico e elágico, taninos e flavonóides glicosilados (Mahmoud et al., 2001), os quais podem ser utilizados na indústria farmacêutica.

Eugenia pluriflora, popularmente conhecida como jabuticaba-do-mato ou papaguela, apresenta propriedades bioativas, pela presença de óleo essencial com ação antifúngica (Souza et al., 2011), especialmente concentrado nos órgãos foliares, de forma abundante. Trata-se de uma espécie indicada para arborização urbana (Lorenzi, 2009), principalmente pelas características do córtex, o qual, frequentemente, apresenta associação com liquens de tonalidades avermelhadas.

A espécie E. pyriformis, também conhecida como uvaia, possui frutos carnosos, frequentemente consumidos in natura, sendo que sua produção já é realizada em escala comercial (Silva et al., 2003). Os frutos de coloração amarelada são ricos em vitamina C (Zillo et al., 2014) e têm sabor ácido a adocicado, ideais para o beneficiamento em forma de sucos, compotas, sorvetes e geleias. O óleo essencial extraído das folhas apresenta flavonóides com propriedades inibidoras $\mathrm{da}$ xantino-oxidase, que atuam no tratamento da gota humana (Theoduloz et al., 1988). Segundo Souza (2013), compostos das folhas e caules dessa espécie possuem potencial antimicrobiano e antifúngico. Seu potencial ecológico está vinculado às interações com a avifauna, $\mathrm{o}$ que torna a espécie recomendável para reflorestamentos heterogêneos destinados à recomposição da vegetação de áreas alteradas em diferentes intensidades (Andrade \& Ferreira, 2000; Gazetta \& Tatto, 2009) e na arborização urbana (Lorenzi, 1998a; Marchiori \& Sobral, 1997).

Algumas espécies de Myrcia apresentam uso medicinal (Rondon et al., 2010), sendo popularmente utilizadas no tratamento de diabetes (Schneider et al., 2008) e indústria farmacêutica. Conhecida popularmente como pitangueira-do-mato ou guamirim, $M$. palustris possui frutos atrativos para a fauna, o que viabiliza seu uso em processos de restauração de ecossistemas alterados. Essa, também, apresenta potencial de uso na arborização urbana (Marchiori \& Sobral, 1997) e, recentemente, estudos realizados por Wubshet et al. (2015) demonstraram que M. palustris é uma importante fonte de fitoquímicos anti-hiperglicêmicos. Dentre os guamirins, o extrato das folhas e óleo essencial de M. guianensis atua como agente alelopático, podendo ser utilizada como herbicida natural (Souza et al., 2006). Nessa já foram encontrados fungos endofíticos com potencial biotecnológico para produção de novos antibióticos (Banhos et al., 2014).

Myrceugenia euosma possui óleos voláteis com altos índices de (E) - nerolidol (Souza et al., 2011; Limberger et al., 2002), o qual age no sistema endócrino humano, sendo eficaz no tratamento de desordens hormonais. Segundo Singh et al. (2005), o ácido morônico isolado dessa espécie possui atividade anti-HIV e a vantagem, frente a medicamentos alopáticos, de apresentar baixa toxicidade. Além do potencial medicinal, possui potencial paisagístico em função da coloração e textura da casca e arquitetura da copa, podendo ser utilizada na arborização urbana (Marchiori \& Sobral, 1997).

A espécie Siphoneugena reitzii possui óleos voláteis com atividade anti-inflamatória (Apel et al., 2002), bem como ação antimicrobiana (Souza et al., 2011). Essas características fazem com que a espécie possa ser utilizada na indústria farmacêutica, alimentícia e cosmética.

O baixo número de espécies da família com potencial frutífero (quatro espécies) ocorre pelo fato de esse potencial ter sido considerado em escala comercial. Algumas Myrtaceae são produzidas em maior escala para suprir a demanda do público consumidor, por exemplo, pitangueira (Eugenia uniflora L.) (Bezerra et al., 2006), jabuticabeira (Plinia spp.) e goiabeira [Acca sellowiana (O. Berg) Burret.] (Ducroquet et al., 2000). Essa última vem sendo cultivada em sistemas agroecológicos no Sul do Brasil e também já foi domesticada em alguns países, como Colômbia e Nova Zelândia. Esse restrito número de Myrtaceae nativas para produção comercial pode ser resultado da falta de estudos, principalmente os vinculados ao melhoramento genético.

Apesar da representatividade das Myrtaceae na Mata Atlântica, estudos específicos ainda são inexpressivos, destacando-se a necessidade de pesquisas sobre melhoramento em produtividade de frutos e manutenção pós-colheita, descoberta de novos componentes químicos dos óleos essenciais, assim como estudos demográficos para embasamento do uso sustentável.

\section{CONCLUSÕES}

Os remanescentes de Floresta Ombrófila Mista presentes na bacia hidrográfica do Rio Caveiras são importantes centros de riqueza das Myrtaceae 
sul-americanas, que em altitudes elevadas ocorrem como população dominante.

O pico de floração e frutificação das Myrtaceae amostradas na bacia hidrográfica do Rio Caveiras ocorre nas estações primavera e verão. Essas Myrtaceae compreendem todas as classificações ecológicas.

Trata-se de uma família botânica indicada para restauração de áreas alteradas, enriquecimento de florestas secundárias, usos ornamentais e fitoterápicos. Apesar da representatividade e potencialidade registradas para as Myrtaceae, ainda são necessárias pesquisas para embasar a conservação das espécies via plano de manejo.

\section{STATUS DA SUBMISSÃO}

Recebido: 23 mar., 2016

Aceito: 3 abr., 2016

\section{AUTOR(ES) PARA CORRESPONDÊNCIA}

\section{Juliano Pereira Gomes}

Departamento de Engenharia Florestal, Universidade do Estado de Santa Catarina UDESC, Avenida Luiz de Camões, 2090, Conta Dinheiro, CEP 88520-000, Lages, SC, Brasil e-mail: julianopgomes@yahoo.com.br

\section{REFERENNCIAS}

Andrade RNB, Ferreira AG. Germinação e armazenamento de sementes de uvaia (Eugenia pyriformis Camb.) Myrtaceae. Revista Brasileira de Sementes 2000; 22(2): 118-125. http://dx.doi.org/10.17801/0101-3122/rbs. v22n2p118-125.

Anjos PJC, Lima AO, Cunha OS, Sousa DP, Onofre ASC, Ribeiro TP et al. Cardiovascular effects induced by linalool in normotensive and hypertensive rats. Zeitschrift fur Naturforschung C. Journal of Biosciences 2013; 68(5-6): 181-190. PMid:23923614. http://dx.doi.org/10.5560/ ZNC.2013.68c0181.

Apel MA, Aleixo A, Suyenaga ES, Chaves CG, Zuanazzi JAS, Limberger RP et al. Antitinflammatory activity of Siphoneugena reitzii (Myrtaceae) and some isolated volatile compounds on chemotaxis of polymorphonuclear leucocytes. Revista de Fitoterapia 2002; 2(1): 303-303.

Backes P, Irgang B. Árvores do Sul: guia de Identificação \& Interesse Ecológico. Porto Alegre: CD Vaz e Ricardo Correa; 2002.
Banhos EF, Souza AQL, Andrade JC, Souza ADL, Koolen HHF, Albuquerque PM. Endophytic fungi from Myrcia guianensis at the Brazilian Amazon: distribution and bioactivity. Brazilian Journal of Microbiology 2014; 45(1): 153-162. PMid:24948926. http://dx.doi.org/10.1590/ S1517-83822014005000027.

Barroso GM, Morim MP, Peixoto AL, Ichaso CLF. Frutos e sementes: morfologia aplicada à sistemática de dicotiledôneas. Viçosa: UFV; 1999.

Bezerra JL, Costa GC, Lopes TC, Carvalho ICDS, Patrício FJ, Sousa SM et al. Avaliação da atividade leishmanicida in vitro de plantas medicinais. Revista Brasileira Farmacognosia 2006; 16: 631-637. http://dx.doi.org/10.1590/S0102695X2006000500008.

Camlofski AMO. Caracterização do fruto de Cerejeira 'Eugenia involocrata DC' visando seu aproveitamento tecnológico [dissertação]. Ponta Grossa: Universidade Estadual de Ponta Grossa; 2008.

Cardoso CAL, Lima ASV, Ré-Poppi N, Vieira MC. Fruit oil of Campomanesia xantocarpa O. Berg and Campomanesia adamantium O.Berg. Journal of Essential Oil Research 2009; 21(6): 481-483. http://dx.doi.org/10.1080/10412 905.2009.9700223

Carvalho AR Jr, Gomes GA, Ferreira RO, Carvalho MG. Constituintes químicos e atividade antioxidante de folhas e galhos de Eugenia copacabanensis Kiaersk (Myrtaceae). Quimica Nova 2014; 37(3): 477-482. http:// dx.doi.org/10.5935/0100-4042.20140079.

Ducroquet JPHJ, Hickel ER, Nodari RO. Goiabeira serrana (Feijoa sellowiana). Jaboticabal: FUNEP; 2000.

Elisabetsky E, Brum LFS, Souza DO. Anticonvulsant properties of linalool in glutamate-related seizure models. Phytomedicine 1999; 6(2): 107-113. PMid:10374249. http:// dx.doi.org/10.1016/S0944-7113(99)80044-0.

Ferreira PI, Gomes JP, Batista F, Bernardi AP, Costa NCF, Bortoluzzi RLC et al. Espécies potenciais para recuperação de áreas de preservação permanente no planalto catarinense. Floresta e Ambiente 2013; 20(2): 173-182. http://dx.doi. org/10.4322/floram.2013.003.

Ferreira PI, Paludo GF, Chaves CL, Bortoluzzi RLC, Mantovani A, Florística E. Fitossociologia arbórea de remanescentes florestais em uma fazenda produtora de Pinus spp. Floresta 2012; 42(4): 783-794. http://dx.doi. org/10.5380/rf.v42i4.21581.

Flora Digital do Rio Grande do Sul. 2015 [citado em 2015 ago. 1]. Disponívem em: http://www.ufrgs.br/fitoecologia/ florars/index.php?pag=result_avanc.php

Gandolfi S, Leitão HF Fo, Bezerra CLE. Levantamento florístico e caráter sucessional das espécies arbustivo arbóreas de uma floresta mesófila semidecídua no município de Guarulhos, SP. Revista Brasileira de Biologia 1995; 55(4): 753-767. 
Gazetta CA, Tatto N. Recuperando as matas ciliares do Vale do Ribeira. São Paulo: Instituto Socioambiental; 2009.

Gressler E, Pizo MA, Morellato LPC. Polinização e dispersão de sementes em Myrtaceae do Brasil. Brazilian Journal of Botany 2006; 29(4): 509-530. http://dx.doi.org/10.1590/ S0100-84042006000400002.

Guzmán-Gutiérrez SL, Gómez-Cansino R, García-Zebadúa JC, Jiménez-Pérez NC, Reyes-Chilpa R. Antidepressant activity of Litsea glaucescens essential oil: identification of E-pinene and linalool as active principles. Journal of Ethnopharmacology 2012; 143: 673-679.

Henriques AT, Sobral M, Bridi R, Vérin P, Menut C, Lamaty $\mathrm{G}$ et al. Essential oils from five southern brazilian species of Myrcia (Myrtaceae). Journal of Essential Oil Research 1997; 9(1): 13-18. http://dx.doi.org/10.1080/1 0412905.1997.9700707.

Higuchi P, Silva AC, Almeida JA, Bortoluzzi RLC, Mantovani A, Ferreira TS et al. Florística e estrutura do componente arbóreo e análise ambiental de um fragmento de Floresta Ombrófila Mista Alto-Montana no município de Painel, SC. Ciência Florestal 2013; 23(1): 153-164. http://dx.doi. org/10.5902/198050988449.

Higuchi P, Silva AC, Ferreira TS, Souza ST, Gomes JP, Silva KM et al. Influência de variáveis ambientais sobre o padrão estrutural e florístico do componente arbóreo, em um fragmento de Floresta Ombrófila Mista Montana em Lages, SC. Ciência Florestal 2012; 22(1): 79-90. http:// dx.doi.org/10.5902/198050985081.

Huo M, Cui X, Xue J, Chi G, Gao R, Deng X et al. Antiinflammatory effects of linalool in RAW 264.7 macrophages and lipopolisaccharide-induced lung injury model. The Journal of Surgical Research 2013; 180(1): 47-54. PMid:22341345. http://dx.doi.org/10.1016/j.jss.2012.10.050.

Kersten RA, Galvão F. Suficiência amostral em inventários florísticos e fitossociológicos. In: Felfili JM, Eisenlohr PV, Melo MMRF, Andrade LA, Meira JAA No. Fitossociologia no Brasil. Viçosa: UFV; 2011.

Köppen W. Climatológia. México: Fondo de Cultura Económica; 1948.

Kuskoski EM, Vega JM, Rios JJ, Fett R, Troncoso AM, Asuero AG. Characterization of anthocyanins from the fruits of Baguaçu (Eugenia umbelliflora Berg). Journal of Agricultural and Food Chemistry 2003; 51(18): 5450-5454. PMid:12926896. http://dx.doi.org/10.1021/jf030014z.

Landrum LR, Kawasaki ML. The genera of Myrtaceae in Brazil: an illustrated synoptic treatment and identification keys. Brittonia 1997; 49(4): 508-536. http://dx.doi. org/10.2307/2807742.

Legrand CD, Klein RM. Mirtáceas: Gomidesia. In: Reitz R. Flora Ilustrada Catarinense. Itajaí: Herbário Barbosa Rodrigues; 1967.
Legrand CD, Klein RM. Mirtáceas: Eugenia. In: Reitz R. Flora Ilustrada Catarinense. Itajaí: Herbário Barbosa Rodrigues; 1969a.

Legrand CD, Klein RM. Mirtáceas: Myrcia. In: Reitz R. Flora Ilustrada Catarinense. Itajaí: Herbário Barbosa Rodrigues; 1969b.

Legrand CD, Klein RM. Mirtáceas: Myrceugenia. In: Reitz R. Flora Ilustrada Catarinense. Itajaí: Herbário Barbosa Rodrigues; 1970.

Legrand CD, Klein RM. Mirtáceas: Calyptranthes. In: Reitz R. Flora Ilustrada Catarinense. Itajaí: Herbário Barbosa Rodrigues; 1971.

Legrand CD, Klein RM. Mirtáceas: Campomanesia, Feijoa, Britoa, Myrrhinium, Hexachlamys, Siphoneugena, Myrcianthes, Neomitranthes, Psidium. In: Reitz R. Flora Ilustrada Catarinense. Itajaí: Herbário Barbosa Rodrigues; 1977.

Legrand CD, Klein RM. Mirtáceas: Myrciaria, Pseudocaryophyllus, Blepharocalyx, espécies suplementares e cultivadas. In: Reitz R. Flora Ilustrada Catarinense. Itajaí: Herbário Barbosa Rodriques; 1978.

Letizia CS, Cocchiara J, Lalko J, Api AM. Fragrance material review on linalool. Food and Chemical Toxicology 2003; 41(7): 943-964. PMid:12804650. http://dx.doi.org/10.1016/ S0278-6915(03)00015-2.

Limberger RP, Apel MA, Sobral M, Moreno PRH, Henriques AT, Menut C. Aromatic plant from Brazil-chemical composition of essencial oils from some Campomanesia species (Myrtaceae). The Journal of Essential Oil Research 2001; 13: 113-115. http://dx.doi.org/10.1080/10412905. 2001.9699630 .

Limberger RP, Simões-Pires CA, Sobral M, Menu C, Bessiere JM, Henriques AT. Essential oils from some Myrceugenia species (Myrtaceae). Flavour and Fragrance Journal 2002; 17(5): 341-344. http://dx.doi.org/10.1002/ffj.1113.

Lorenzi H. Árvores brasileiras: manual de identificação e cultivo de plantas arbóreas nativas do Brasil. Nova Odessa: Plantarum; 1998a.

Lorenzi H. Árvores brasileiras: manual de identificação e cultivo de plantas arbóreas nativas do Brasil. Nova Odessa: Plantarum; 1998b.

Lorenzi H. Árvores brasileiras: manual de identificação e cultivo de plantas arbóreas nativas do Brasil. Nova Odessa: Plantarum; 2009.

Lughadha EN, Proença C. A survey of the reproductive biology of the Myrtoideae (Myrtaceae). Annals of the Missouri Botanical Garden 1996; 83(4): 480-503. http:// dx.doi.org/10.2307/2399990.

Mahmoud II, Marzouk MAS, Moharram FA, El-Gindi MR, Hassan AMK. Acylated flavonol glycosides from Eugenia jambolana leaves. Phytochemistry 2001; 58(8): 1239-1244. PMid:11738415. http://dx.doi.org/10.1016/ S0031-9422(01)00365-X. 
Marchiori JNC, Sobral M. Dendrologia das angiospermas: Myrtales. Santa Maria: UFSM; 1997.

Martins DR, Chaves CL, Bortoluzzi RLC, Mantovani A. Florística de floresta ombrófila mista Altomontana de Campos em Urupema, Santa Catarina, Brasil. Revista Brasileira de Biociências 2011; 9: 156-166.

Park SN, Lim YK, Freire MO, Cho E, Jin D, Kook J. Antimicrobial effect of linalool and $\alpha$-terpineol against periodontopathic and cariogenic bacteria. Anaerobe 2012; 18(3): 369-372. PMid:22537719. http://dx.doi. org/10.1016/j.anaerobe.2012.04.001.

R Development Core Team. R: a language and environment for statistical computing. Vienna; 2010.

Reitz R. Plano de coleção. In: Reitz R, editor. Flora Ilustrada Catarinense. Itajaí: Herbário Barbosa Rodrigues; 1965.

Rio Grande do Sul. Governo do Estado. Secretaria Estadual do Meio Ambiente. Relatório Final do Inventário Florestal Contínuo do Rio Grande do Sul. Porto Alegre: SEMA; 2001.

Romagnolo MB, Souza MC. Os gêneros Calycorectes O. Berg, Hexachlamys O. Berg, Myrcianthes O. Berg, Myrciaria O. Berg e Plinia L. (Myrtaceae) na planície alagável do alto rio Paraná, Brasil. Acta Botanica Brasílica 2004; 18(3): 613-627. http://dx.doi.org/10.1590/S010233062004000300019 .

Rondon RM No, Santos JS, Silva MA, Loppe VC. Potencialidades de uso de espécies arbustivas e arbóreas em diferentes fisionomias de Cerrado, em Lucas do Rio Verde/MT. Revista de Biologia e Ciências da Terra 2010; 10(2): 113-126.

Schneider NFZ, Moura NF, Colpo T, Marins K, Marangoli C, Flach A. Estudo dos compostos voláteis e atividade antimicrobiana da Myrciaria tenella (cambuí). Revista Brasileira de Farmacologia 2008; 89(2): 131-133.

Silva CV, Bilia DA, Maluf AM, Barbedo CJ. Fracionamento e germinação de sementes de Uvaia (Eugenia pyriformis Cambess. - Myrtaceae). Revista Brasileira de Botanica. Brazilian Journal of Botany 2003; 26(2): 213-221. http:// dx.doi.org/10.1590/S0100-84042003000200009.

Singh IP, Sandip BB, Bhutani KK. Anti-HIV natural products. Current Science 2005; 89(2): 269-290.
Sobral M, Proença C, Souza M, Mazine F, Lucas E. Myrtaceae in Lista de Espécies da Flora do Brasil. 2016 [citado em 2016 out. 18]. Disponível em: http://floradobrasil.jbrj. gov.br/jabot/floradobrasil/FB10335

Sobral MA. Família Myrtaceae no Rio Grande do Sul. São Leopoldo: Unisinos; 2003.

Souza AM. Avaliação do potencial antimicrobiano de Eugenia pyriformis Cambess., Myrtaceae e estudo da Associação Sinérgica com agentes antibacterianos e antifúngicos de uso clínico [dissertação]. Curitiba: Universidade Federal do Paraná; 2013.

Souza APS Fo, Santos RA, Santos LS, Guilhon GMP, Santos AS, Arruda MSP et al. Potencial alelopático de Myrcia guianensis. Planta Daninha 2006; 24(4): 649-656.

Souza GHB, Mello JCP, Lopes NP. Farmacognosia: Coletânea Científica. Ouro Preto: Universidade Federal de Ouro Pedro; 2011.

The Plant List. Myrtaceae [online]. 2013 [citado em 2015 set. 22]. Disponível em: http://www.theplantlist.org/1.1/ browse/A/Myrtaceae

Theoduloz C, Franco L, Ferro EB, Schmeda Rarschmann G. Xanthine oxidase inhibitory activity of Paraguayan Myrtaceae. Journal of Ethnopharmacology 1988; 24(2-3): 179-183. PMid:3253488. http://dx.doi.org/10.1016/03788741(88)90149-3.

Torres MR. Compilación y análisis sobre los productos forestales no madereros (PFNM) en el Perú. San Tiago: FAO; 2001.

Wubshet SG, Moresco HH, Tahtah Y, Brighente IM, Staerk D. High-resolution bioactivity profiling combined with HPLC-HRMS-SPE- NMR: a-Glucosidase inhibitors and acetylated ellagic acid rhamnosides from Myrcia palustris DC. (Myrtaceae). Phytochemistry 2015; 116: 246-252. PMid:25935545. http://dx.doi.org/10.1016/j. phytochem.2015.04.004.

Zillo RR, Silva PPM, Zanatta S, Spoto MHF. Parâmetros físico-químicos e sensoriais de polpa de uvaia (Eugenia Pyriformis) submetidas à pasteurização. Bioenergia em Revista: Diálogos 2014; 4(2): 20-33. 